\title{
Neue Strategien bei der elektronischen Datenerfassung in klinischen Studien: endlich Vorteile auch für die Prüfärzte
}

\author{
Z. Dobbie, C. Bernasconi
}

\section{Zusammenfassung}

Die elektronische Erfassung klinischer Studiendaten im Prüfzentrum (Electronic Data Capture) hat gegenüber den klassischen «Papierstudien» anerkannte Vorteile für die Pharmaindustrie. In diesem Artikel legen wir die Hauptvorteile der neu entwickelten Systeme für das Studienteam im Prüfzentrum dar: bessere Übersicht dank schneller Einsicht in die zuletzt gesammelten Daten, einfachere Dateneingabe, weniger Rückfragen (Queries), freiere Arbeitsweise und Reduktion der Monitoringvisiten. Ausserdem diskutieren wir wesentliche Punkte für die Entscheidung, ob sich ein Prüfzentrum bei einer klinischen Studie auf die elektronische Datenerfassung einlassen sollte.

\section{Einleitung}

Seit etwa zehn Jahren beschäftigt sich die pharmazeutische Industrie mit der elektronischen Erfassung von Studiendaten im Prüfzentrum (sogenanntes Remote Data Entry oder Electronic Data Capture, EDC). Die Vorteile der EDC-Strategie für die Pharmaindustrie (Sponsoren) sind anerkannt: hohe Transparenz dank sofortiger Verfügbarkeit der gesammelten Daten, Möglichkeit, auf Vorkommnisse schneller zu reagieren, d. h. auch höhere Sicherheit der Studienbehandlung, höhere Qualität der primären Daten dank direkter Eingabechecks. Ausserdem ergibt sich aus diesen Faktoren eine Reduktion der Studienkosten. Das Ziel dieses Artikels ist aufzuzeigen, dass vor allem aufgrund ausgereifter Technologie (z.B. Internetstabilität) und progressiver Konzeptverbesserung heutzutage der Benefit von EDC-Systemen auch für die Prüfzentren wesentlich sein kann.

\section{Hybrid- und Online-EDC-System}

Elektronische Datenerfassung bedeutet, dass im Prüfzentrum erhobene Studiendaten elektronisch erfasst und direkt in die zentrale Datenbank übertragen werden. Zwei verschiedene Typen von EDC-Systemen werden heutzutage in klinischen Studien eingesetzt, wobei der Unterschied zwischen ihnen gerade für das Prüfzentrumsteam grosse Implikationen hat [1].

In Hybridsystemen (On-/Offline, Web-enabled) werden in einer ersten Phase die Eingaben ins eCRF (electronic Case Report Form, elektronischer Prüfbogen) lokal im Prüfzentrum gespeichert; in einer zweiten Phase erfolgt die Datenübertragung in die zentrale Studiendatenbank, meist via Internet. Die Datenzwischenspeicherung im Prüfzentrum verlangt entsprechend ausgebaute Prozesse vor Ort: Installation, Validierung und Verwaltung der studienspezifischen Software wie auch Implementierung von Sicherheitsmassnahmen gegen Datenverlust und Datendiebstahl.

Die Alternative zu diesem zweiphasigen Prozess ist die direkte Dateneingabe vom Prüfzentrum in die zentrale Datenbank durch die sogenannten Online-Systeme (Web-based). Dabei werden eCRF-Seiten über einen üblichen Internetbrowser (z.B. Internet Explorer, Netscape Navigator) ausgefüllt. Die Studiendaten werden zu keinem Zeitpunkt auf dem lokalen Rechner gespeichert. Es wird keine Software installiert und validiert und jegliche Rechner im Prüfzentrum mit einem Internetzugang können für die Dateneingabe benutzt werden.

\section{Datenschutz und Datensicherheit}

Bestimmte Datenschutzgesetze, die z.B. die Anonymisierung der Patientendaten verlangen, sind sowohl bei EDC- wie auch bei Papierstudien gleichermassen anwendbar. Bei EDC-Studien entfallen sämtliche für das Handling der Papierprüfbogen relevanten Sicherheitsmassnahmen. Andererseits erfordern spezifische EDC-Richtlinien (z. B. der FDA 21 CFR part 11 [2]), dass die EDC-Systeme die Datensicherheit sowohl beim Speichern als auch bei der Datenübertragung garantieren. Die stark verschlüsselte Datenüber- 
Tabelle 1

Was soll der Prüfarzt fragen?

Infrastruktur im Prüfzentrum
- Hardware vorhanden? Falls nein, wird sie vom Sponsor zur Verfügung gestellt?
- Internetzugang vorhanden?
- Möglichkeit, online zu arbeiten vorhanden?
Know-how im Prüfzentrum
- IT-Kenntnisse erforderlich?
- EDC-Training gewährleistet?
- Softwaremanual vorhanden?
- Help-line (für IT und wissenschaftliche Fragen) vom Sponsor gewährleistet?
EDC-System
- Online- oder Hybridsystem?
- Demoversion zur Beurteilung der Benutzerfreundlichkeit und Geschwindigkeit des Systems
verfügbar?
- Automatisches Einspeisen der elektronisch vorhandenen Daten in die Datenbank möglich?
- Direkte Eingabe der Laborwerte durch das Zentrallabor möglich?
- Elektronisches Querymanagement vorhanden?
Honorarzahlung
- Zahlungsplan der Prüfärztehonorare abhängig von der Vollständigkeit der Dateneingabe?

\section{Vorteile für den Prüfarzt im Studien- management}

Das EDC-System, als Plattform für den Informationsaustausch zwischen allen Studienbeteiligten, ermöglicht einen zeit- und ortsunabhängigen Zugriff auf alle relevanten Studiendaten und -unterlagen. Dank eines professionellen eCRFDesigns wird die Dateneingabe im Vergleich zu Papierstudien bedienungsfreundlicher und effizienter. Interaktive Anweisungen, fertige Eingabevorschläge, z.B. in Form von Pull-downMenüs, automatische Datenüberprüfung und Hinweise auf mögliche Eingabefehler resultieren in einer höheren Qualität der eingegebenen Daten. Die Folge ist eine Verminderung von Rückfragen (Queries) an den Prüfarzt (bis zu 80\% weniger [4]), was eine erhebliche Zeitersparnis für ihn bedeutet. Ausserdem verläuft die Bearbeitung der Rückfragen elektronisch innerhalb des EDC-Systems und somit unabhängig z.B. von Monitorbesuchen, was die Freiheit bei der Arbeitsgestaltung fördert. Zusammen mit der Tatsache, dass die Anzahl der Monitorbesuche reduziert werden kann (auch wenn diese für die Qualitätssicherung im Prüfzentrum prinzipiell bestehenbleiben müssen), führt dies zu einer Vereinfachung der Teilnahmemöglichkeit von kleineren oder peripheren Prüfzentren an grossen, multizentrischen Studien [5].

Weiterhin ist es in manchen Fällen möglich, Patientendaten, die bereits elektronisch im Prüfzentrum vorhanden sind (z. B. Labordaten oder elektronische Patiententagebücher), automatisch in das EDC-System einzuspeisen [6]. Dieser Aspekt kann eine erhebliche Zeitersparnis und eine hohe Datenqualität bedingen.

Weitere praktische Vereinfachungen ergeben sich aus einem automatisierten System für die Medikamentenlieferung und einem verlässlichen System für die rechtzeitige und korrekte Zahlung der Prüfarzthonorare.

\section{Bedenken und Diskussionspunkte seitens des Prüfarztes}

Bei der Umstellung von Papierstudien auf elektronische sind die Prüfzentren unter den meistherausgeforderten. Die technischen Voraussetzungen sind bei den Online-EDC-Systemen auch ohne intensive Unterstützung seitens IT-Abteilungen in Spitälern relativ einfach erfüllt; die modernsten EDC-Softwarelösungen gewährleisten mit einer herkömmlichen analogen Netz-
6 Jacobson J. Sites and EDC: Making EDC Site-Friendly. EDC-Today, Issue 5, 1-5. tragung über das Internet (128 Bit «high enwort ermöglicht dessen selektive Führung d das EDC-System. Jedem Benutzer wird eine Rolle Systems der Benutzer Zugriff hat und zu we linien sollte das EDC-System über eine ss nannte Änderungskontrolle (Data Audit Trail) verfügen, die zu jedem beliebigen Zeitpunkt teneingabe und eventuellen Datenänderungen m System zu rekonstruieren.

ihre Passworte streng vertraulich behandeln, bleibt ein Unsicherheitsfaktor im Bereich der Datensicherheit vorhanden. Man darf allerdings nicht übersehen, dass auch Papiersysteme keinesfalls uneingeschränkt sicher sind, und dass die Wahrscheinlichkeit, dass Papierdaten verlorengehen, gestohlen oder für eine nicht berechtigte Person zur Einsicht gestellt werden, erheblich ist. 
7 Hellsten D. What to ask EDC vendors. Applied Clinical Trials 2001;10(5):2-4

8 Friedman R. Widening the web. GCP Journal 2003;10(3):17-8. verbindung eine komfortable Arbeitsgeschwindigkeit. Die technische Limitierung des OnlineEDC-Systems zeigt sich eventuell erst beim Transfer von Files mit sehr grossen Datenmengen (z.B. Bilddaten).

Für eine reibungslose Umstellung im Prüfzentrum wird eine Schulung aller Beteiligten im Zentrum essentiell. Es muss weiterhin sichergestellt werden, dass für die Prüfärzte jederzeit ein Ansprechpartner in der Studienzentrale erreichbar ist (Help-Desk).

In der heutigen Übergangsphase werden dem Prüfarzt von Sponsoren für die Durchführung klinischer Studien häufig beide Lösungen angeboten, Papier und EDC. In der Tabelle 1 sind diejenigen Punkte aufgeführt, die der Prüfarzt bezüglich EDC beachten sollte, bevor er sich für eine der Alternativen entscheidet [siehe auch 7].

\section{Plädoyer}

Trotz aller Vorteile eines Online-EDC-Systems, das schätzungsweise heutzutage in über 500 klinischen Studien weltweit eingesetzt wird [8], wird momentan die Mehrheit aller klinischen Studien mit Papier-CRFs durchgeführt [2]. Der Hauptgrund sind die seit Jahren etablierten Prozesse, die auf die Verwendung von auf Papier basierten Systemen abgestimmt sind. Beim Studiensponsor sowie auch im Prüfzentrum benötigt die Umstellung zu eCRFs Änderungen in Personalstrukturen und Betriebsorganisationen und erfordert generell eine neue Einstellung der involvierten Personen. Es bleibt die Aufgabe der Sponsoren, durch ausreichende Aufklärung die nötige Bereitschaft und Motivation bei den Prüfärzten zu erzielen. 